\title{
Impacts of worldwide individual non-pharmaceutical interventions on COVID-19 transmission across waves and space
}

\author{
Yong Ge ${ }^{\text {a,b, 1,* }}$, Wen-Bin Zhang ${ }^{\text {a,b,1 }}$, Haiyan Liu ${ }^{\text {c,1 }}$, Corrine W Ruktanonchai ${ }^{\text {d,e, }}$, Maogui Hu ${ }^{\text {a,b }}$, \\ Xilin $\mathrm{Wu}^{\mathrm{a}}{ }^{\mathrm{a}}$, Yongze Song ${ }^{\mathrm{f}}$, Nick W Ruktanonchai ${ }^{\mathrm{d}, \mathrm{e}}$, Wei Yan ${ }^{\mathrm{g}, 1}$, Eimear Cleary ${ }^{\mathrm{e}}$, \\ Luzhao Feng ${ }^{\mathrm{h}}$, Zhongjie $\mathrm{Li}^{\mathrm{i}}$, Weizhong Yang ${ }^{\mathrm{i}}$, Mengxiao Liu ${ }^{\mathrm{a}}{ }^{\mathrm{b}}$, Andrew J Tatem ${ }^{\mathrm{e}}$, \\ Jin-Feng Wang ${ }^{\mathrm{a}, \mathrm{b}}$, Shengjie Lai ${ }^{\mathrm{e}, \text { " }}$ \\ ${ }^{a}$ State Key Laboratory of Resources and Environmental Information System, Institute of Geographic Sciences and Natural Resources Research, Chinese Academy of \\ Sciences, Beijing, China \\ ${ }^{\mathrm{b}}$ College of Resources and Environment, University of Academy of Sciences, Beijing, China \\ ${ }^{\mathrm{c}}$ Marine Data Center, South Marine Science and Engineering Guangdong Laboratory (Zhuhai), Zhuhai, China \\ ${ }^{\mathrm{d}}$ Population Health Sciences, Virginia Tech, Blacksburg, VA, USA \\ ${ }^{\mathrm{e}}$ WorldPop, School of Geography and Environmental Science, University of Southampton, UK \\ ${ }^{\mathrm{f}}$ School of Design and the Built Environment, Curtin University, Perth, 6101, Australia \\ ${ }^{\mathrm{g}}$ Respiratory Medicine Department, Peking University Third Hospital, Beijing, China \\ ${ }^{\mathrm{h}}$ School of Population Medicine and Public Health, Chinese Academy of Medical Sciences \& Peking Union Medical College, Beijing, China \\ ${ }^{\mathrm{i}}$ Divisions of Infectious Diseases, Chinese Center for Disease Control and Prevention, Beijing, China
}

\section{A R T I C L E I N F O}

\section{Keywords:}

COVID-19

Big data

Non-pharmaceutical interventions

Multi-scale

Effectiveness

\begin{abstract}
A B S T R A C T
Governments worldwide have rapidly deployed non-pharmaceutical interventions (NPIs) to mitigate the COVID19 pandemic. However, the effect of these individual NPI measures across space and time has yet to be sufficiently assessed, especially with the increase of policy fatigue and the urge for NPI relaxation in the vaccination era. Using the decay ratio in the suppression of COVID-19 infections and multi-source big data, we investigated the changing performance of different NPIs across waves from global and regional levels (in 133 countries) to national and subnational (in the United States of America [USA]) scales before the implementation of mass vaccination. The synergistic effectiveness of all NPIs for reducing COVID-19 infections declined along waves, from $95.4 \%$ in the first wave to $56.0 \%$ in the third wave recently at the global level and similarly from $83.3 \%$ to $58.7 \%$ at the USA national level, while it had fluctuating performance across waves on regional and subnational scales. Regardless of geographical scale, gathering restrictions and facial coverings played significant roles in epidemic mitigation before the vaccine rollout. Our findings have important implications for continued tailoring and implementation of NPI strategies, together with vaccination, to mitigate future COVID-19 waves, caused by new variants, and other emerging respiratory infectious diseases.
\end{abstract}

\section{Introduction}

The COVID-19 pandemic has caused significant disruption to daily lives, causing over 184 million confirmed cases and 4 million deaths as of 4 July 2021 (WHO, 2021). Non-pharmaceutical interventions (NPIs) have been deployed across the World to curb the pandemic (Perra, 2021). With the rollout of COVID-19 vaccines using different dosing and population targeting strategies (Saad-Roy et al., 2021), robust vaccination programs would enable the relaxation of NPIs (ECDC, 2021; Huang et al., 2021a). However, given the delays in vaccine production and the inequality of vaccine allocations (Zhou, 2020) as well as the emergence of novel variants (Kupferschmidt, 2021; Lai et al., 2021a), NPIs should be maintained to avoid further resurgences before herd immunity can be achieved (ECDC, 2021; Pang et al., 2020; Yang et al., 2021).

The impact of NPI policies might be dynamic, determined by a variety of factors such as policy fatigue and population immunity. First, because of variations in the government's execution of NPIs and the degree of people's inclination to comply, the same NPI may work

\footnotetext{
* Corresponding authors.

1 These authors contributed equally to this work.
} 
differently in different regions. Second, over a lengthy period adopting NPIs, people tend to experience psychological tiredness, reducing the effectiveness of NPIs (Goldstein et al., 2021). Third, changes in mutant viruses, vaccination rates, and immunity acquired from infections may have an impact on the efficacy of individual NPIs (De Visscher et al., 2021). Given that there is a long way to go before herd immunity for COVID-19 (Aschwanden, 2021) is achieved, understanding the role of different NPIs in reducing COVID-19 transmission before vaccine rollouts is critical for tailoring effective NPI strategies for future COVID-19 waves and other epidemics caused by respiratory infections.

The effectiveness of NPIs on pandemic mitigation had been demonstrated by previous studies that mostly focused on the first wave of the pandemic (Baker et al., 2021; Flaxman et al., 2020; Huang et al., 2021a; Lai et al., 2020a; Li et al., 2021; Tian et al., 2020), with limited analysis of subsequent waves and multi-scale research (Hale et al., 2021b). The implementation of NPIs in the first wave had, to some degree, changed human knowledge and perceptions, behaviours and responses to mitigate the outbreaks (Doogan et al., 2020; Farooq et al., 2020; Geldsetzer, 2020; Hu et al., 2020; Brauner et al., 2021). The enduring importance of NPI responses to COVID-19 has also been highlighted (Moore et al., 2021). Though policy fatigue has been proposed and confirmed in the implementation of NPIs (Crane et al., 2021), whether, and to what extent, NPI effectiveness decreases with fatigue in subsequent waves remains unclear. Additionally, the effects of NPIs may vary across countries, nations and subnational regions with different geographical characteristics, such as health capacity, residential population density, aging ratio, humidity and air temperature (Hsiang et al., 2020; Van Bavel et al., 2020; Zhang et al., 2021; Ge et al., 2021). The potential differences in NPI effectiveness across multi-geographical levels are rarely discussed in existing analyses (Li et al., 2021).

In this study we estimated the effects of several individual NPIs as well as their combinations by identifying their contributions to the decay ratio of COVID-19 infections across waves at different geographical levels before the onset of any vaccination program. Based on the big data collected from multiple public available datasets, we assembled a national database, covering 133 countries, territories and areas, to estimate NPI efficacy at both global and regional scales, and a subnational database, covering 51 states of the United States of America, to evaluate national and subnational NPI efficacy in the USA. The effects of NPIs across wave and space can provide localized insights into tailoring effective NPI strategies for future COVID-19 waves.

\section{Material and methods}

\subsection{Data sources and processing}

\subsubsection{Epidemiological data}

The daily number of confirmed cases reported by country were obtained from the COVID-19 Data Repository by the Center for Systems Science and Engineering (CSSE) at Johns Hopkins University (JHU) (Dong et al., 2020). While the state-level cases for the US were reported by the CDC (Prevention, 2021). The cases were recorded after infectionto-confirmation delay since the onset of their infection. To remove the influence of outliers and the fluctuation caused by the day-of-week effect, we smoothed daily case counts with the Gaussian kernel by calculating the rolling average using a Gaussian window with a standard deviation of 2 days, truncated at a maximum window of 15 days (Haug et al., 2020).

\subsubsection{Intervention policy data}

The non-pharmaceutical interventions studied in this work were collected and generated from the Oxford COVID-19 Government Response Tracker (OxCGRT) (Hale et al., 2021a). The global and regional analysis studied school closures, workplace closures, public transport closures, gathering restrictions, international travel restrictions, movement restrictions and facial coverings. With respect to the national and subnational context, we replaced international travel control and movement restrictions with internal movement restrictions and stay-at-home orders, respectively, due to the changing of collinearity and rare variation in international travel control across states of the US. The intensity of NPIs policies documented in OxCGRT was scaled into discrete values between 0 and 1 by dividing their maximum intensity, where 0 represented an absence of the NPI and 1 represented the corresponding maximum intensity. The intensity of school closures was further corrected as 1 during public and school holidays (Lai et al., 2020b).

\subsubsection{Environmental and demographic covariates}

To control for country-specific confounders in the estimates of intervention effectiveness varied across countries, we also assembled population density, aging ratio, health capacity index, air temperature, and humidity for all these 133 study countries. Within each country, population density (per square kilometre) was the ratio of the total population over the corresponding built-up area in 2014 (Florczyk et al., 2019). The total and age-grouping population data in 2019 were obtained from the United Nations to calculate the aging ratio ( $>65$ year old) among populations (Nations, 2019). Health capacity index was the arithmetic average of the five indices, including i) prevent, ii) detect, iii) respond, iv) enabling function, and v) operational readiness, developed to characterize the health security capacities in the context of the COVID-19 outbreak (Kandel et al., 2020). Air temperature and humidity were derived from the Global Land Data Assimilation System (Rodell et al., 2004). With respect to state-level data of the US, we used an alternative health capacity index, i.e., bed capacity, to capture the uneven distribution of hospital capacity relative to regional need, as well as substantial geographic variation in bed capacity per capita from 2012 (Care, 2021).

To further remove the day-of-week effect among case testing, diagnosis, and data reporting, all data used in this study were assembled and aggregated into a weekly dataset. The studied countries were selected by being documented in every dataset of epidemiological data, intervention policy data and environmental and demographic covariates. The details of data collection and processing are further provided in the Supplementary Information.

\subsection{Defining waves and groups}

\subsubsection{Waves}

The inequality in pandemic development across the world has led some countries to confront more than one COVID-19 wave (Aleta et al., 2020; Kuehn, 2021). To identify potential variation in effects of NPIs across waves, we divided the epidemic waves in each country/state based on the smoothed daily reported cases. An epidemic wave constituted a period of three or more consecutive weeks in each country/state, when the daily numbers of cases within this period all higher than $5 \%$ of the maximum daily number of cases in 2020 in corresponding countries/ states. The first and last days of these defined time periods were the start and end of the corresponding wave, respectively. Noting that the first wave of the pandemic in most countries/states began with low-level community transmission caused by imported cases, we adjusted the start date of the first wave. It was set to the day when the number of daily new cases exceeded 10 cases for countries where the maximum number of daily new cases in the first wave were no $>300$ cases. Otherwise, the start date was set to the day when the number of daily new cases exceeded 20 cases. The details and full lists of waves by country/state can be found in SI. Up to now, no more than three waves of epidemics have been detected before the implementation of mass vaccination in most countries/states.

\subsubsection{Regional stratification}

The reported COVID-19 morbidity and mortality showed obvious spatial stratified heterogeneity among different countries/states, based 
on the released epidemiological data. A spatial variance analysis method known as a geographical detector model (Wang and $\mathrm{Xu}$, 2017; Wang et al., 2016) was used to divide the study countries/states into different groups, according to each country/state's overall morbidity and mortality during the whole research period. Under the circumstance, the groups were also implicitly divided by the testing rate (see SI Section C.2). Spatial proximity was also considered within groups because nearby countries/states were prone to have similar policies, intervention methods, as well as environmental conditions. We investigated the spatial variation in NPIs effectiveness by dividing 133 countries into four groups at the regional level and 51 states into three groups at the subnational level based on their COVID-19 morbidity and mortality together with geographical proximity (Fig. 1). Thresholds of 1,800 per 100,000 persons for morbidity and 40 per 100,000 persons for mortality were determined by q-statistic index in the geographical detector model to select countries with both high morbidity and high mortality. Considering the geographical proximity between countries, Asian countries and African countries were stratified into two separate groups.

\subsection{Model description}

A Bayesian inference model (Brauner et al., 2021; Flaxman et al., 2020) was built to disentangle the individual effects of NPIs from the empirical changes of weekly growth rates. We measured relative contributions of NPIs on the observed decay ratio of COVID-19 infections (denoted as $\% \Delta \omega_{t}$ ) using the existence and intensity change of these interventions.

$\omega_{t}=\omega_{0} \prod_{i=1}^{n} \exp \left(-\left(\alpha_{i}+\sigma_{i}\right) x_{i, t}\right)+\varepsilon$,

where $\omega_{0}$ represents the baseline growth rate without interventions, $\alpha_{i}$ is the coefficient of NPIs and control variables $x_{i, t}$ on day $t$, and $\varepsilon$ is the error term representing the uncertainty of decay ratio. The confounding variables were linearly added into the model regarding the NPI vari- ables. In contrast to the control variables used to describe the countryspecific difference in NPIs efficacy, we also introduced normal error term $\sigma_{i}$ for $x_{i}$ to capture the intrinsic variation of effectiveness across countries. The effect of NPIs set $X$ in a period, such as the first wave of the pandemic, can be interpreted as a decay ratio in $\omega_{0}$ by computing $e_{i}=1-\prod_{x_{i} \in X} \exp \left(-\alpha_{i} \bar{x}_{l}\right)$, where $\bar{x}_{l}$ is the average strength of the NPI $x_{i}$ during that period. The highest effect of NPIs set $X$ is 1 , representing that the transmission is fully contained or interrupted by the set.

The decay ratio was defined as a percentage of reduction in the baseline growth rate by the instantaneous growth rate. The instantaneous growth rate of transmission at each point of time was calculated as the current weekly number of new infections over the infections in the previous week. In addition to interventions, there were many other factors (e.g., the transmissibility of new variants and the variation of case diagnosis and reporting) that might affect the growth rate of COVID-19 transmission over time. Therefore, the baseline growth rates in different waves and countries were assumed as the mean of the top three highest instantaneous growth rates in the corresponding wave and country. We used the mean of highest growth rates to increase the stability of the baseline and model outputs, as the rate calculated from empirical data might be easily affected by an unusually high single value of reported case number.

We used the spatiotemporal Bayesian inference model to evaluate the effect coefficients in Eq. (1) based on the observed real-time COVID19 growth rates, identifying the relative NPIs and vaccination effectiveness. We first evaluated the global effectiveness of NPIs for the whole data context (Fig. 2). In addition to the overall NPIs effectiveness, we also evaluated NPIs regional effects in the first, second and third waves for each country group to show the potential large spatiotemporal diversity, respectively. Finally, national and subnational variation of NPIs efficacy in space and time was demonstrated by the case of the USA with 51 states. To exclude the vaccination impact on COVID-19 growth, we only used data before the onset of the vaccination project in all countries. All estimations were performed using Markov chain Monte Carlo
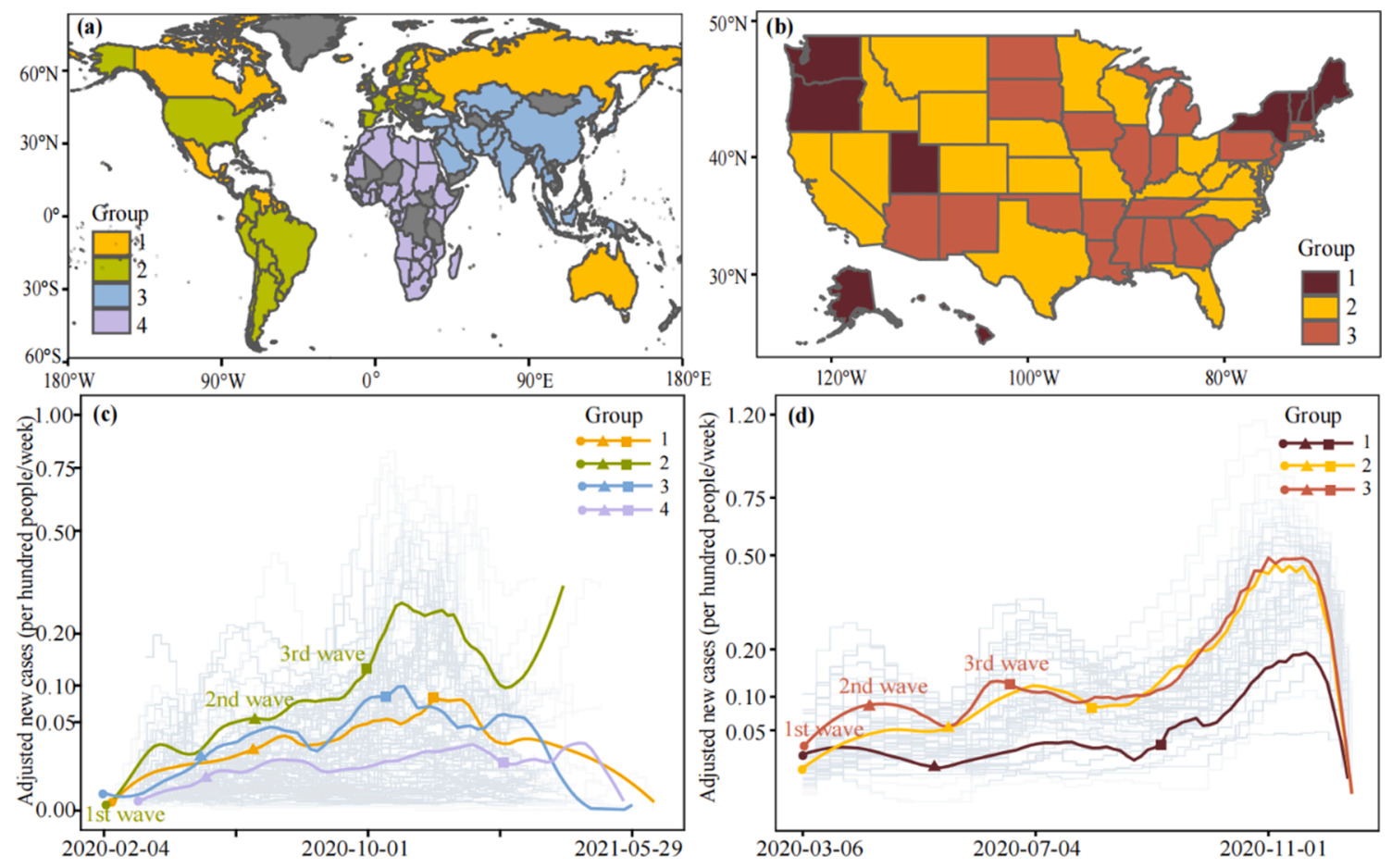

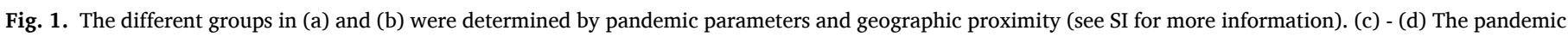

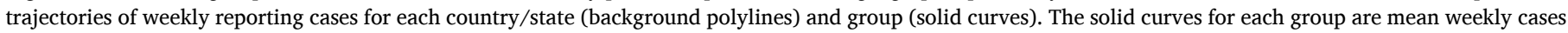

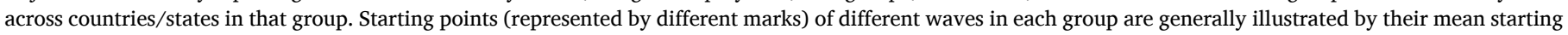

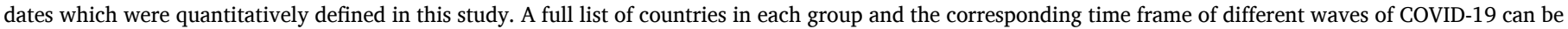
found in SI Table C1 - C4, C6 - C8. 


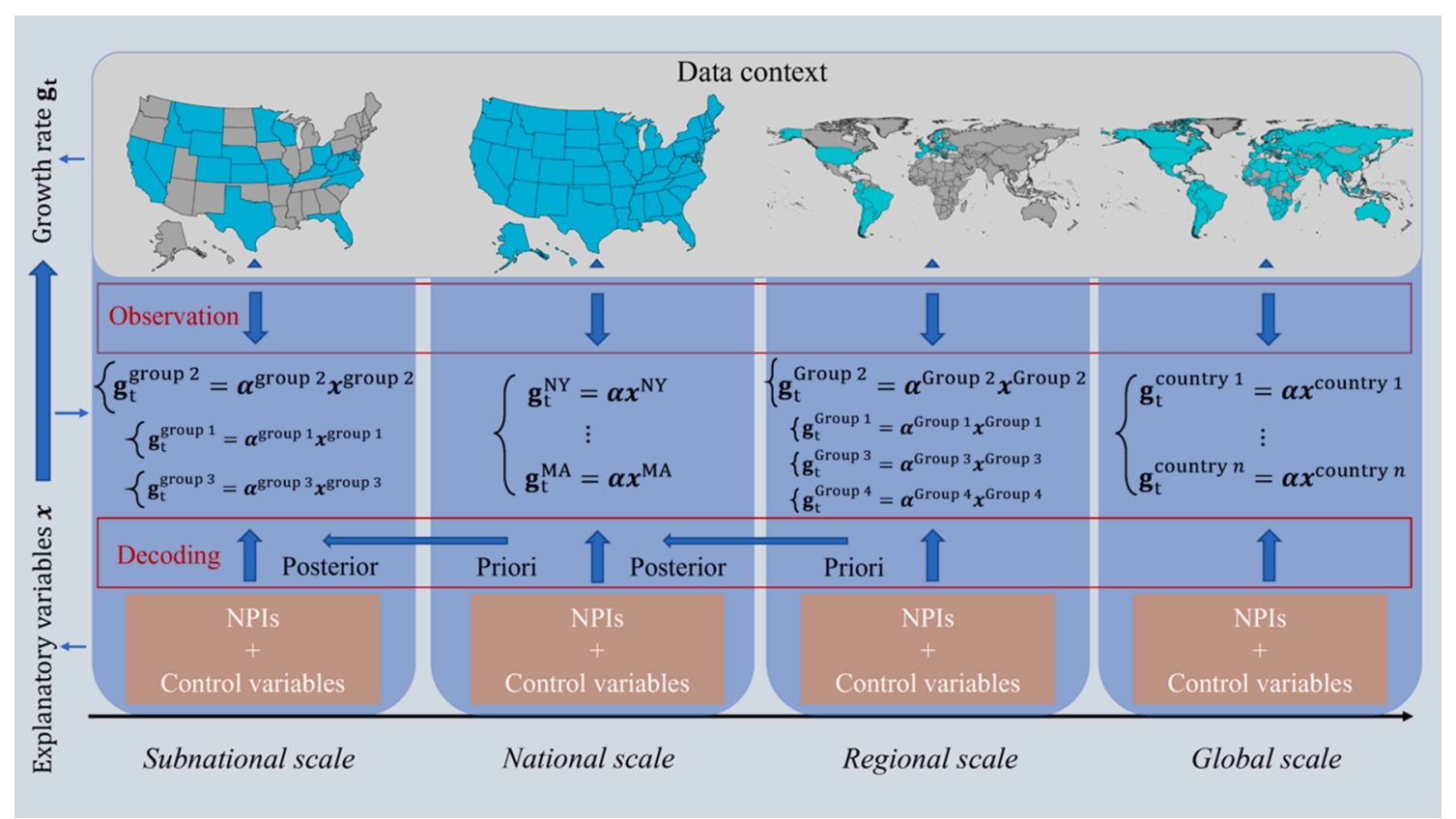

Fig. 2. The computational environmental settings for analysis from subnational scale to global scale. We used growth rate as the outcome variable to describe the trajectory of the pandemic. The empirical changes of growth rate were decoded into the effectiveness of both NPIs as well as the control variables.

(MCMC) methods. The reliability of our model was assessed by the crossvalidation for overall intervention effects. Sensitivity analyses were also performed to assess model robustness in terms of our assumptions.

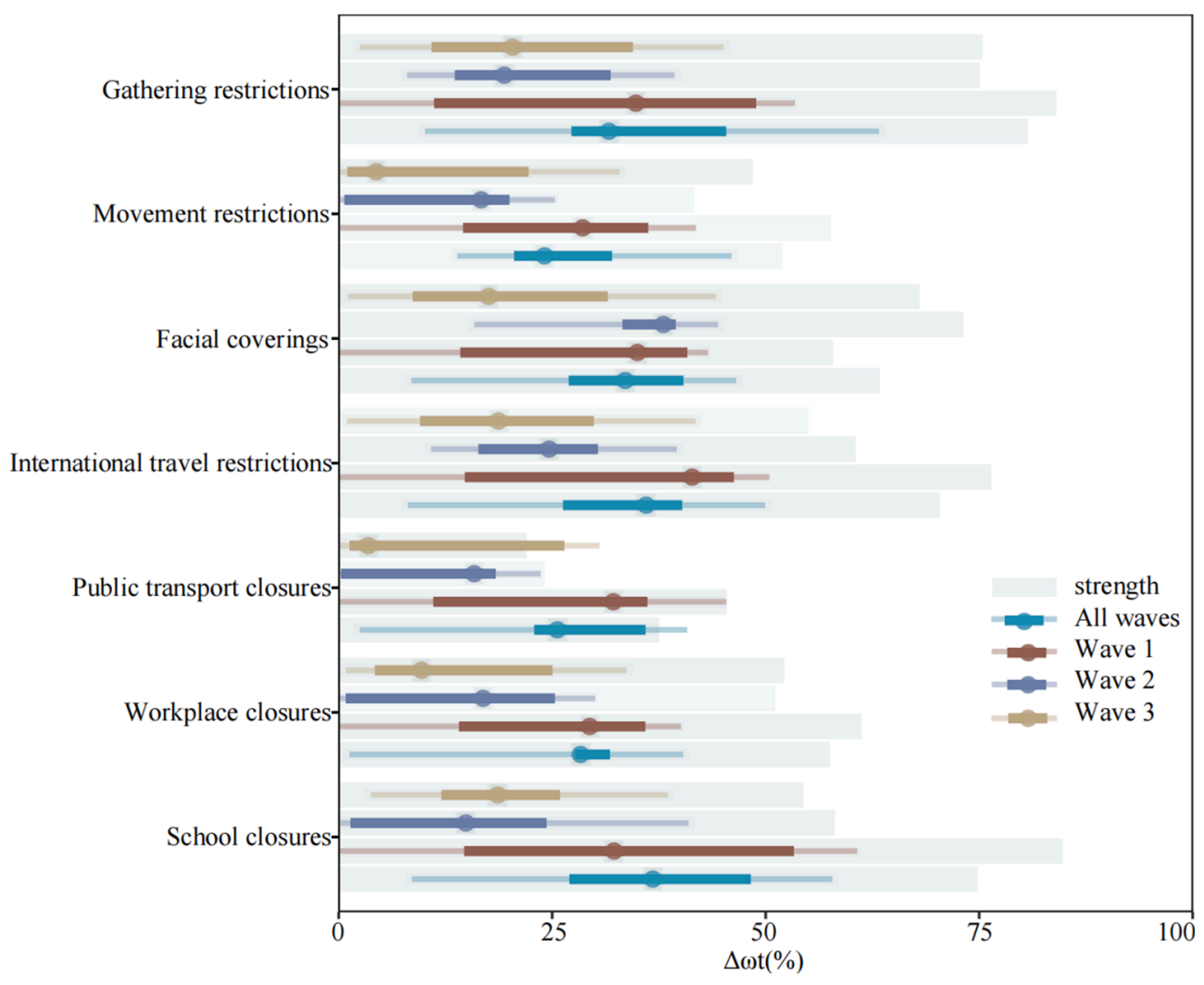

Fig. 3. Effects of individual NPIs on reducing the transmission of COVID-19 across waves within our data context. The coefficients $\left(\alpha_{i}\right)$ of NPIs parameters in different periods were calibrated by the default model setting with corresponding data contexts. The effect estimates were calculated by the coefficients of NPIs through $1-\exp \left(-\alpha_{i} \overline{x_{i}}\right)$, where $\overline{x_{i}}$ is the average strength of NPI implementation (represented by the background shadow). We rescaled the average strength by multiplying 100 to adapt the $\mathrm{x}$-axis. The synergistic effectiveness of all NPIs (All waves: $92.3 \%$, Wave 1: $95.4 \%$, Wave 2: $79.9 \%$, Wave 3: $56.0 \%$ ) were nonlinear cumulative in terms of the individual effect by $1-\prod_{x_{i}} \exp \left(-\alpha_{i} \overline{x_{i}}\right)$. The effect over all waves represents the average performance of NPIs against COVID-19 in 133 countries (Fig. 1(a)) before their vaccination by 22 June 2021. Wave 1 refers to the average performance of NPIs against COVID19 in the first wave of the 133 countries. The specific periods of the first wave in 133 countries are not fully consistent, meaning that the first wave does not refer to a particular time but a general period of the first outbreak. The second wave refers to the periods starting from the second outbreak. $\%$ $\Delta \omega_{t}$ represents a decay ratio of the COVID-19 infection rate in each country. The 5th, 25th (Q1), 50th (median), 75th (Q3), and 95th percentiles of estimates of $\% \Delta \omega_{t}$ are presented to indicate details of the variations. The uncertainty intervals of NPI effectiveness refer to the variance over corresponding data contexts. 


\section{Results}

\subsection{Global effects of individual NPIs across waves}

We estimated all mitigation strategies of immediate interest before the start of mass vaccination implementation, where the four NPIs with the highest impacts $(>30 \%)$ on transmission growth rate included school closures (median $36.8 \%$, interquartile range [IQR] 27.0-48.3\%), international travel restrictions $(36.0 \%, 26.3-40.2 \%)$, facial coverings (33.6\%, 27.0-40.4\%) and gathering restrictions $(31.7 \%, 27.2-45.4 \%)$ (Fig. 2). The NPIs with moderate effects $(25 \%-30 \%)$ included workplace closures $(28.3 \%, 27.7-31.8 \%)$ and public transport closures $(25.6 \%, 22.9-35.9 \%)$, while movement restrictions had relatively limited impacts. The overall synergistic effectiveness of these NPIs reached $92.3 \%$ (IQR: 88.1-96.9\%) and declined with the epidemic process of COVID-19, from $95.4 \%$ in the first wave to $56.0 \%$ in the third wave.

The effectiveness and dominance of NPIs varied across waves (Fig. 3). In the first wave, the most effective NPI was international travel restrictions (median 41.4\%, IQR 14.8-46.3\%). Results also showed that the synergistic effectiveness of all NPIs exceeded $28 \%$ in the first wave. In the second wave, facial coverings became the NPI with the highest effect $(38.0 \%, 33.2-39.5 \%)$, while gathering restrictions became the most effective NPI in the third wave $(20.4 \%, 10.9-34.5 \%)$. In addition, the effects of workplace closures, public transport closures and movement restriction declined to 9.7 (IQR 4.2-25.4\%), 3.5\% (1.3-26.4\%) and $4.41 \%(1.0-22.2 \%)$ in the third wave, respectively.

\subsection{Regional NPIs impacts across waves by country group}

Further, this study revealed that effects of individual nonpharmaceutical measures showed discernible spatial and temporal variations across countries and waves, when only the periods before vaccine rollouts were included for accurate evaluation of NPIs (Fig. 4).

In the first wave, gathering restrictions in group 3 had the highest contribution (median 52.5\%, IQR 30.5-58.6\%) to transmission reduction. We found all NPIs in Group 3, i.e., Asian countries, were generally more effective than other groups for the first wave, especially gathering restrictions $(52.5 \%, 30.5-58.6 \%)$, school closures $(45.7 \%, 36.4-54.6 \%)$ and facial coverings (43.7\%, 35.4-44.8\%). In Group 4, all NPIs showed moderate effects $(>20 \%)$, with the exception of public transport closure (17.4\%, 11.8-22.6\%).

In the second wave, four NPIs in Group 1 had the highest effectiveness among four country groups. Facial coverings had an important role in reducing transmission for group 1 from the first to the second wave $\left(\% \Delta \omega_{t}>30 \%\right)$ and behaved similarly in the second Group's second-to- third waves. Gathering restrictions and school closures exerted a relatively strong effect in both groups during the first two waves $(>20 \%)$. International travel restrictions were one of the most effective NPI in affecting the pandemic transmission of Group 1 and made a notable contribution (median $33.52 \%$, IQR $23.82-41.91 \%$ ) in the second wave. In Group 4, effects of NPIs were limited ( $<7 \%$ ), except for facial coverings $(41.3 \%, 37.6 \%-44.9 \%)$ and school closures $(13.0 \%, 9.4 \%-$ $17.8 \%)$.

After the first wave, as interventions were gradually relaxed, the effectiveness of most individual NPIs had declined by different degrees in different country groups. This decline was mainly observed in Group 2, i.e., the European, American, and Oceanian countries with relatively high morbidity and mortality, and Group 3, i.e., Asian countries. Countries in Group 1 and Group 2 were generally comprised of European, American, and Oceanian countries with relatively low and high morbidity and mortality, respectively. The highest effects of all NPIs in different waves were $97.9 \%$ for Group 3 in the first wave, $89.2 \%$ for Group 1 in the second wave, and $69.3 \%$ for Group 2 in the third wave. From the first to the third wave, effects of most individual NPIs were reduced, apart from facial coverings in Group 2 and school closures in Group 4, whose effects were increased. Workplace closures always played a mild role in controlling the spread of the virus in Group 2 for all waves but had limited effectiveness for Group 1 in the third wave.

In the third wave, effects of most NPIs had been critically reduced. The effectiveness of workplace closures (from $31.2 \%$ to $6.0 \%$ in median), public transport closures (from $33.2 \%$ to $5.8 \%$ ) and movement restrictions (from $33.0 \%$ to $4.9 \%$ ) declined from the first to the third wave. International travel restrictions were the only NPI which had stable effectiveness in all waves $(>15 \%)$ for Group 3 . Distinguishable from other groups, in Group 4, i.e., African countries, five out of seven NPIs had the lowest effectiveness in the second wave and then climbed in the third wave, resulting in the joint effect of all NPIs in the third wave surpassing that in the second one. Facial coverings (median $41.3 \%$, IQR $37.6-44.9 \%)$ of the second wave and school closures $(30.8 \%$, $21.8-36.6 \%$ ) of the third wave were the only NPIs in this group which surpassed other groups in suppressing infection.

\subsection{National and subnational effectiveness of NPIs in the USA}

This study used the USA, as well as its States, as prisms to explain the potential spatio-temporal heterogeneity of NPI efficacy on a national and subnational scale, respectively. Similar to results at the global scale, the overall synergistic effectiveness of NPIs showed a downward trend as the COVID-19 pandemic spread across the USA, from $83.27 \%$ (IQR $82.06-84.56 \%)$ in the first wave to $58.74 \%(57.01-60.33 \%)$ in the third wave, of which gathering restrictions and stay-at-home orders had the

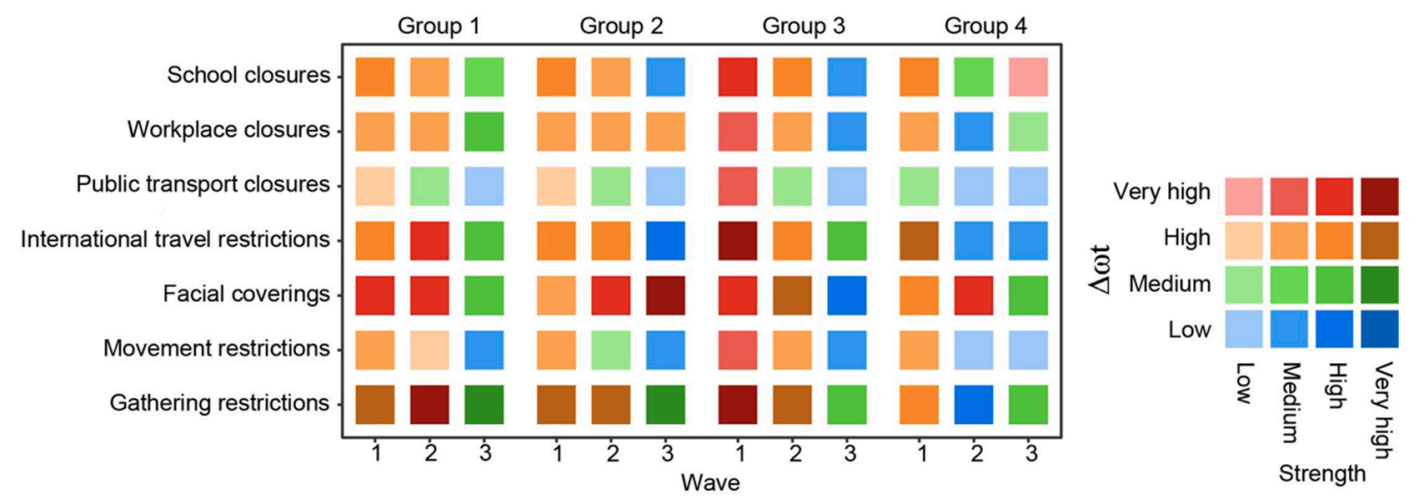

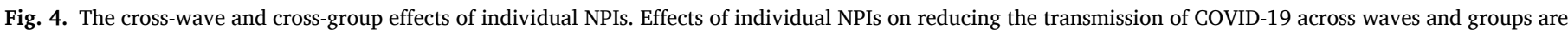

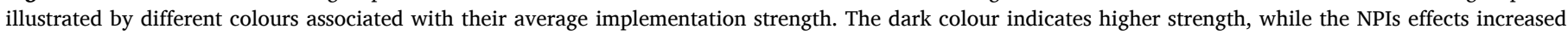

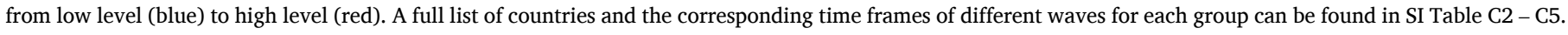
(For interpretation of the references to colour in this figure legend, the reader is referred to the web version of this article.) 
highest effect ( $>15 \%$ ) on mitigating outbreaks (Fig. 5). NPIs which had moderate impact were facial coverings and school closures $(>10 \%)$, while public transport closures and workplace closures had limited effectiveness $(<5 \%)$.

From a temporal perspective, the variability of NPIs was assessed through comparison of effects of NPIs across State groups. In the first wave, school closure was the primary NPI that was associated with a significant decline in incidence $(>40 \%)$. The association between school closures and incidence increased with morbidity and mortality among groups. Regardless of school closures, the other six NPIs with lower effectiveness were internal movement restrictions (median 33.9\%, IQR $28.3-43.5 \%)$, workplace closures $(25.5 \%, 18.4-32.8 \%)$ and gathering restrictions (19.1\%, 13.5-25.0\%) in Group 1. In Group 2, gathering restrictions had the highest effectiveness (59.3\%, 54.8-63.3\%) beneath school closures $(64.5 \%, 58.8 \%-69.4 \%)$, while facial coverings $(14.5 \%$, $12.7-16.2 \%)$ worked in parallel with internal movement restrictions $(14.1 \%, 10.8-17.3 \%)$ and gathering restrictions $(14.5 \%, 11.3-17.9 \%)$ in Group 3. In the second wave, school closures still had the highest effectiveness in controlling transmission in Group 1. Facial coverings significantly enhanced the effectiveness in Group 1, from $4.9 \%$ $(3.2-7.2 \%)$ in the first wave to $37.7 \%(32.3-42.5 \%)$ in the second wave. In addition, stay-at-home orders had similar contributions, from $2.2 \%$ (1.4-3.3\%) to $25.8 \%(20.3-31.0 \%)$ in Group 2 and $2.6 \%(1.7-3.9 \%)$ to $31.2 \%(25.6-36.3 \%)$ in Group 3. In the third wave, primary NPIs included facial coverings $(24.4 \%, 16.2-32.8 \%)$ and school closures $(22.8 \%, 15.8 \%-29.8 \%)$ in Group1; gathering restrictions $(21.1 \%$, $15.3-26.7 \%)$ and stay-at-home orders $(20.7 \%, 15.7 \%-25.4 \%)$ in Group; and stay-at-home orders (14.5\%, 9.8-19.5\%), school closures (13.8\%, 8.9\% - 19.3\%), facial coverings $(12.0 \%, 7.8 \%-17.1 \%)$, internal movement restrictions $(10.3 \%, 6.6 \%-14.7 \%)$, and gathering restrictions $(9.0 \%, 5.6 \%-13.5 \%)$ in Group 3.

\subsection{Model validation}

The reliability of our models and corresponding results were evaluated by the leave-forty-countries-out cross validation. We first calibrated our model using $70 \%$ countries (93 countries), randomly selected from 133 countries, to estimate the overall NPIs effects in both the first and second waves. Then, we derived the instantaneous growth rates through the estimated overall effects of NPIs for the remaining 30\% countries (40 countries) in terms of their implemented interventions. We used mean square error, ranging from 0 to infinite with 0 representing the perfect prediction ability, to assess the difference between the predicted instantaneous growth rates and the corresponding empirical instantaneous growth rates. We repeated this procedure 50 times, where the average mean square error was (median 1.4, interquartile range [IQR] 1.3 - 2.0). Further, we standardised the predicted and empirical instantaneous growth rates, respectively, within each country and then analysed all the data with one-way ANOVA.

\subsection{Sensitivity analysis}

The robustness of models and parameters used in the study was also assessed by a series of sensitivity analyses. The parameters to be assessed included: i) the probability mass of NPIs and vaccination on negative effectiveness and ii) the probability mass of sociodemographic factors on negative effectiveness. In this study, the default values for these parameters were $20 \%$ and $50 \%$, respectively. The comparison of parameter impacts on estimates were listed in SI Table B1, representing three scenarios with smaller and larger default parameter settings. The differences of NPI effects among three waves were tested using a Wilcoxon signed-rank test, a non-parametric statistical hypothesis test for comparing NPIs effects between pairs of the three waves.

\section{Discussion}

Based on longitudinal public health interventions and sociodemographic datasets across COVID-19 waves, our study revealed that NPI measures played overwhelming roles in mitigating the pandemic, with varied effects across multi-spatial and temporal scales. Before the implementation of mass vaccination, the effectiveness of each individual NPI had been over $24.1 \%$ on the global level, $10.7 \%$ on the regional level, $1.0 \%$ on the USA national level, and $1.2 \%$ on the USA subnational level. Regardless of geographic scale and pandemic wave, the overall impact of integrated NPIs had been over $52.9 \%$ before the start of mass vaccination. Our results presented individual and synergistic NPI effectiveness in global, regional, national, and subnational scales, and this study was the first impact assessment that extended the research period from the beginning of the epidemic to before the vaccine rollout, to our knowledge. These findings are crucial for continued tailoring and implementation of NPI strategies to mitigate COVID-19 transmission among future waves (e.g., because of new variants of concern) or similar emerging infectious diseases, such as pandemic influenza.

On a global level, the synergistic effectiveness of NPIs had been declining along the waves before the start of mass vaccination. Lockdown fatigue or pandemic-policy fatigue might be the main reason for this phenomenon. Previous studies have found pandemic-policy fatigue to be geographically widespread, based on self-reported behaviours from a million respondents (Crane et al., 2021; Petherick et al., 2021). Reported adherence to high-cost and sensitising interventions, like movement restrictions, decreased, while reported adherence to low-cost and habituating interventions, like facial coverings, increased in 2020. In our findings, the global efficiency of movement restrictions declined from $28.56 \%$ (IQR $14.56-36.25 \%$ ) in the first wave to $16.67 \%$ $(0.65-20.00 \%)$ in the second wave, while that of facial coverings climbed from $34.98 \%(14.24-40.82 \%)$ to $38.03 \%$ (33.22-39.48\%), which is in accordance with the emergence of pandemic-policy fatigue. Our results show that the synergistic impact of NPIs and even the effect of individual facial coverings in the recent third wave was less than that in

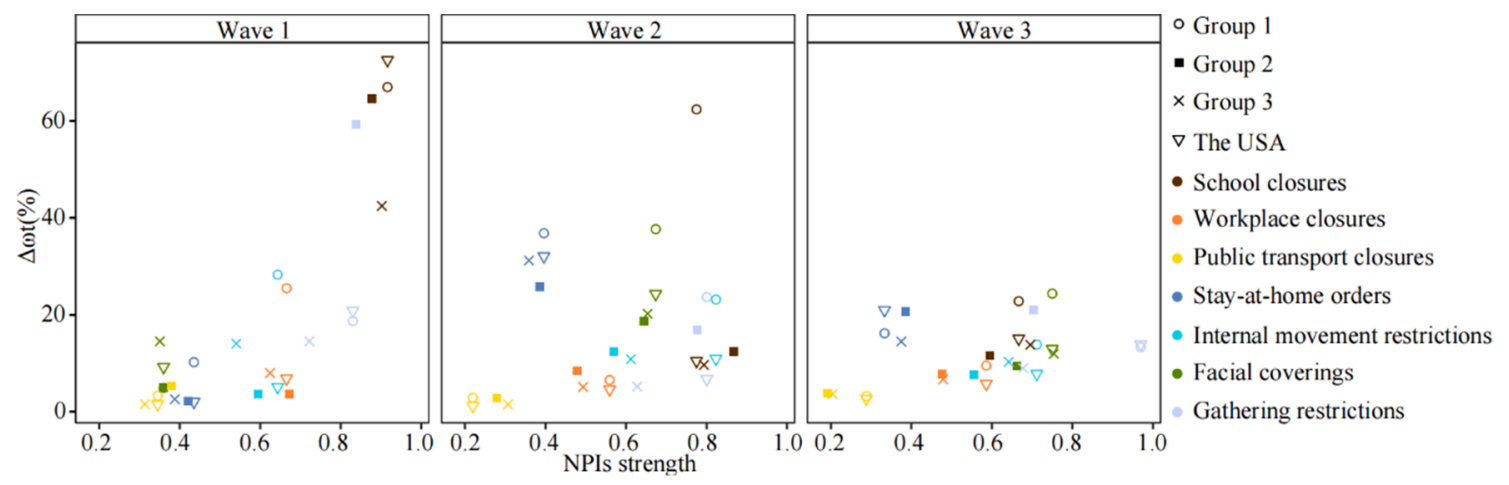

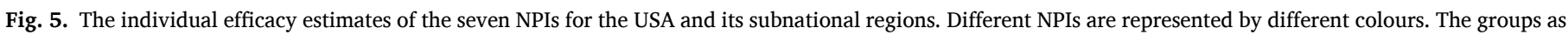

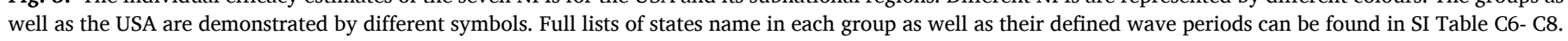


the second, suggesting the fatigue might continue to increase before vaccine rollout. Fatigue might have spread from high-cost interventions to lost-cost ones. Therefore, emphasizing the importance of policy compliance may have to be put on the agenda by local policy makers in pandemic mitigation.

The synergistic effectiveness of NPIs was not found to decrease among all groups and across all waves, however. In Group 1, i.e., American, and Oceanian countries with relatively low morbidity and mortality, the integrated efficiency of NPIs peaked at the second wave. These countries, except for Russia that only had one wave in our analysis, were largely protected from the epidemic due to the international travel control in the first wave (Lai et al., 2021b), and witnessed an increased infection rate from $0.52 \%$ in the first wave to $0.73 \%$ in the second wave. The increased infection rate tends to push local people to react to the outbreak and maintain vigilance, bringing a higher synergistic NPI effect in the second wave than that in the first one. In Group 2 and Group 3, i.e., American, and Oceanian countries with relatively high morbidity and mortality, and Asian countries, respectively, the synergistic impact of NPIs decreased with each wave. They both experienced the most violent epidemic attack in the first wave, and thereafter, a better understanding of the coronavirus reduced people's anxiety about the epidemic. Due to economic reasons and people's expectations of recovery, the implementation strength of NPIs declined from average of 0.67 in the first wave to 0.63 in the third wave in Group 2, and from 0.69 to 0.52 in Group 3, further reducing the effect of NPIs. For Group 4, African countries, though the infection rate declined along the waves, the efficiency of integrated NPIs in the third wave surpassed that in the second wave. It is worth noting that many Africa nations might lack reliable epidemic data because of their reluctance to acknowledge epidemic or inadequate testing due to poverty and conflict (Nguimkeu and Tadadjeu, 2021). Under-reporting confirmed case numbers and under-updated policy data would bring great uncertainty to the analysis results. Our results prove that the groups have different epidemic development and epidemic prevention trajectories. Countries should consider their own epidemic trajectory when learning from other countries' experience in epidemic prevention and control.

There is partial consistency in NPI effectiveness at multiple geographical scales. Firstly, gathering restrictions and facial coverings both played significant roles in epidemic mitigation at a global scale and the USA national scale. The significant effects of gathering restrictions may be attributed to transmission most commonly occurring through spread of the virus in droplets or aerosols among people in close contact (Jayaweera et al., 2020). Therefore, appropriate levels of gathering restrictions and facial coverings should be maintained in subsequent waves before herd immunity is achieved. Secondly, the effect of school closures in epidemic control was significant at both the global and national level, with substantial heterogeneity between waves and geographic areas at both a regional and subnational level. The strongest effect of school closure was observed during the first wave at both global and the USA national level, as well as Group 3 at the regional level and Group 1 at the USA subnational level. School closures included not only primary and second level education institutes, but also universities which may serve as a bridge population for family/community transmission of the coronavirus (Head et al., 2021). In areas where school closures have had a good effect on epidemic control, this NPI can still be used during the vaccine delivery stage. Thirdly, international travel restrictions had a stronger protective effect than movement restrictions at both a global and regional level, while movement restrictions, especially stay-at-home orders, reflected its protective role at the national and subnational scale. Countries that quickly implemented border controls might have reduced the seeding of COVID-19 between countries, but international travel restrictions cannot prevent local transmission at the community level in countries where the virus had already been introduced. The increased effect of movement restriction on infection transmission, contributed to by both stay-at-home orders and internal movement restrictions in our study, at national and subnational level proved that our model has a good explanatory power for the effect of NPIs on epidemic migration.

It should be noted that our research limited the study period from the start of case reporting to the start of vaccination in each country. Therefore, pandemic-policy fatigue which happened before the vaccination rollout might worsen in the vaccination era. However, studies have shown that vaccination alone is insufficient to contain the outbreak, even with the most optimistic assumption of $85 \%$ infection prevention of vaccines (Moore et al., 2021). Especially for the poor and people living in areas with low resources (Zhou, 2020). Nonpharmaceutical interventions should therefore not be rapidly relaxed in the vaccination era, considering the emergence of new variants and the inequality of vaccine delivery among countries (Huang et al., 2021b).

We acknowledge that there are limitations in our analysis. First, data collected from public data sources may generate certain uncertainty. We did not make our own datasets from the sources but used publicly released ones from Johns Hopkins University, CDC, OxCGRT, United Nations, AHA, and so on. Data produced by different institutions may have differences in data due to subtle differences in statistical calibres and related regulations. Due to the huge amount of data and the relatively reliable data sources, in the absence of obvious inconsistencies in the data, we did not analyse the differences in data source statistics and uncertainty, which might bring uncertainties to the results. Second, the interactions among the seven NPIs were not considered in this study, and it is difficult to identify the effects of individual interventions and define the causality between NPIs and the change of Covid-19 trajectories if countries implemented several NPIs simultaneously (Flaxman, et al., 2020; Sharma et al., 2021). However, the uncoordinated deployed NPIs strategies across countries might have provided an opportunity to evaluate the empirical effectiveness of NPIs over space and times, and such data-generated correlations between NPIs implementation and Covid-19 transmission have provided important insights of NPI effectiveness in the first wave (Brauner, et al., 2021; Flaxman, et al., 2020). Third, we used grouped regional research instead of researching each country while the effects of NPIs might have some differences in different countries. However, the group results should be more robust than that from a single country because of a larger number of samples in each group considered. The effects of NPIs within each group were assumed following gamma distribution based on previous studies (Flaxman et al., 2020; Lai et al., 2020a). The obtained different NPI effects reflected the overall effects in different waves and groups while varied in different countries.

Overall, the disclosure of epidemic, publicized responses and big data allow us to estimate and compare the cross-wave effects of public health measures at global, regional, national, and subnational scales. Our work provides a quantitative basis and approach to explore historic spatio-temporal variation in the effectiveness of individual NPIs before the mass implementation of vaccination. The overall effectiveness of NPIs shows a downward trend between waves, possibly due to policy fatigue. Even though the synergistic efficiency of NPIs has been over $50 \%$ in recent waves before the vaccine rollout, the reducing effect over time deserves our vigilance, especially in areas lacking vaccines. Through the verification of multi-scale results, our study certified the effectiveness of gathering restrictions and facial coverings in the epidemic mitigation, which could be maintained in the following waves and mitigate pandemics caused by other emerging respiratory infectious diseases in the future.

\section{Data and code availability}

All source code and data necessary for the replication of our results and figures are available at: https://github. $\underline{\text { com/wxl1379457192/NPIs_code }}$ 


\section{Declaration of Competing Interest}

The authors declare that they have no known competing financial interests or personal relationships that could have appeared to influence the work reported in this paper.

\section{Acknowledgments}

This study was supported by the National Natural Science Foundation of China (Grant No. 41725006 and 81773498), the Bill \& Melinda Gates Foundation (INV-024911 and OPP1134076), and the Beijing Natural Science Foundation (Grant No. 7212128).

\section{Author contributions}

YG, WBZ, HYL, WY, CWR and SJL conceived and designed the study, built the model, collected data, finalised the analysis, interpreted the findings, and wrote the manuscript. MGH, XLW, YZS and MXL collected data, interpreted the findings, and revised drafts of the manuscript. NWR, EC, JFW, LF, ZJL, WZY and AJT interpreted the findings, and commented on and revised drafts of the manuscript. All authors read and approved the final manuscript.

\section{Ethical approval}

Ethical clearance for collecting and using secondary data in this study was granted by the institutional review board of the University of Southampton (No. 61865). All data were supplied and analysed in an anonymous format, without access to personal identifying information.

\section{Role of the funding source}

The funder of the study had no role in study design, data collection, data analysis, data interpretation, or writing of the report. The corresponding authors had full access to all the data in the study and had final responsibility for the decision to submit for publication. The views expressed in this article are those of the authors and do not represent any official policy.

\section{Appendix A. Supplementary material}

Supplementary data to this article can be found online at https://doi. org/10.1016/j.jag.2021.102649.

\section{References}

Aleta, A., Martin-Corral, D., y Piontti, A.P., Ajelli, M., Litvinova, M., Chinazzi, M., Dean, N.E., Halloran, M.E., Longini Jr, I.M., Merler, S., 2020. Modelling the impact of testing, contact tracing and household quarantine on second waves of COVID-19. Nat. Hum. Behav. 4, 964-971.

Aschwanden, C., 2021. Five reasons why COVID herd immunity is probably impossible. Nature 591, 520-522.

Baker, R.E., Yang, W., Vecchi, G.A., Metcalf, C., Grenfell, B.T., 2021. Assessing the influence of climate on wintertime SARS-CoV-2 outbreaks. Nat. Commun. 12.

Brauner, J.M., Mindermann, S., Sharma, M., Johnston, D., Salvatier, J., Gavenčiak, T., Stephenson, A.B., Leech, G., Altman, G., Mikulik, V., 2021. Inferring the effectiveness of government interventions against COVID-19. Science 371.

Care, T.D.A.o.H., 2021. Bed Capacity.

Crane, M.A., Shermock, K.M., Omer, S.B., Romley, J.A., 2021. Change in reported adherence to nonpharmaceutical interventions during the COVID-19 pandemic, April-November 2020. JAMA 325, 883-885.

De Visscher, A., Sutton, B., Sutton, T., 2021. Second-wave Dynamics of COVID-19: Impact of Behavioral Changes, Immunity Loss, New Strains, and Vaccination.

Dong, E., Du, H., Gardner, L., 2020. An interactive web-based dashboard to track COVID 19 in real time. Lancet. Infect. Dis 20, 533-534.

Doogan, C., Buntine, W., Linger, H., Brunt, S., 2020. Public perceptions and attitudes towards COVID-19 non-pharmaceutical interventions across six countries: a topic modeling analysis of twitter data (preprint). J. Med. Internet Res. 22.

ECDC, 2021. Integrated COVID-19 response in the vaccination era.

Farooq, A., Laato, S., Islam, A., 2020. Impact of online information on self-isolation intention during the COVID-19 pandemic: cross-sectional study. J. Med. Int. Res. 22
Flaxman, S., Mishra, S., Gandy, A., Unwin, H., Walker, P.G., 2020. Estimating the effects of non-pharmaceutical interventions on COVID-19 in Europe. Nature.

Florczyk, A.J., Corbane, C., Ehrlich, D., Freire, S., Kemper, T., Maffenini, L., Melchiorri, M., Pesaresi, M., Politis, P., Schiavina, M., 2019. GHSL data package 2019. Luxembourg, EUR 29788, 290498.

Geldsetzer, P., 2020. Knowledge and perceptions of COVID-19 among the general public in the United States and the United Kingdom: a cross-sectional online survey. Ann. Intern. Med. 173, 157-160.

Ge, Y., Zhang, W.B., Wang, J., Liu, M., Ren, Z., Zhang, X., Zhou, C., Tian, Z., 2021. Effect of different resumption strategies to flatten the potential COVID-19 outbreaks amid society reopens: a modeling study in China. BMC Public Health 21 (1), 1-10.

Goldstein, P., Yeyati, E.L., Sartorio, L., 2021. Lockdown fatigue: The diminishing effects of quarantines on the spread of COVID-19.

Hale, T., Angrist, N., Goldszmidt, R., Kira, B., Petherick, A., Phillips, T., Webster, S., Cameron-Blake, E., Hallas, L., Majumdar, S., 2021a. A global panel database of pandemic policies (Oxford COVID-19 Government Response Tracker). Nat. Hum. Behav. 1-10.

Hale, T., Angrist, N., Hale, A.J., Kira, B., Majumdar, S., Petherick, A., Phillips, T., Sridhar, D., Thompson, R.N., Webster, S., 2021b. Government responses and COVID19 deaths: Global evidence across multiple pandemic waves. PLoS ONE 16, e0253116.

Haug, N., Geyrhofer, L., Londei, A., Dervic, E., Desvars-Larrive, A., Loreto, V., Pinior, B., Thurner, S., Klimek, P., 2020. Ranking the effectiveness of worldwide COVID-19 government interventions. Nat. Hum. Behav. 4, 1303-1312.

Head, J.R., Andrejko, K.L., Cheng, Q., Collender, P.A., Phillips, S., Boser, A., Heaney, A. K., Hoover, C.M., Wu, S.L., Northrup, G.R., 2021. School closures reduced social mixing of children during COVID-19 with implications for transmission risk and school reopening policies. J. Royal Soc. Interface 18, 20200970.

Hsiang, S., Allen, D., Annan-Phan, S., Bell, K., Bolliger, I., Chong, T., Druckenmiller, H., Huang, L.Y., Hultgren, A., Krasovich, E., 2020. The effect of large-scale anticontagion policies on the COVID-19 pandemic. Nature 584, 262-267.

Hu, Z., Lin, X., Kaminga, A.C., Xu, H., 2020. Impact of the COVID-19 epidemic on lifestyle behaviors and their association with subjective well-being among the general population in Mainland China: Cross-sectional study. J. Med. Internet Res. 22.

Huang, B., Wang, J., Cai, J., Yao, S., Lai, S., 2021a. Integrated vaccination and physical distancing interventions to prevent future COVID-19 waves in Chinese cities. Nat. Hum. Behav. 5, 695-705.

Huang, J., Liu, X., Zhang, L., Zhao, Y., Wang, D., Gao, J., Lian, X., Liu, C., 2021b. The oscillation-outbreaks characteristic of the COVID-19 pandemic. Natl. Sci. Rev.

Jayaweera, M., Perera, H., Gunawardana, B., Manatunge, J., 2020. Transmission of COVID-19 virus by droplets and aerosols: A critical review on the unresolved dichotomy. Environ. Res. 188, 109819.

Kandel, N., Chungong, S., Omaar, A., Xing, J., 2020. Health security capacities in the context of COVID-19 outbreak: an analysis of International Health Regulations annual report data from 182 countries. The Lancet 395, 1047-1053.

Kuehn, B.M., 2021. Africa Succeeded Against COVID-19's First Wave, but the Second Wave Brings New Challenges. JAMA 325, 327-328.

Kupferschmidt, K., 2021. Viral mutations may cause another 'very, very bad'COVID-19 wave, scientists warn. Science.

Lai, S., Floyd, J., Tatem, A., 2021a. Preliminary risk analysis of the spread of new COVID19 variants from the UK. South Africa and Brazil.

Lai, S., Ruktanonchai, N.W., Carioli, A., Ruktanonchai, C.W., Floyd, J.R., Prosper, O., Zhang, C., Du, X., Yang, W., Tatem, A.J., 2021b. Assessing the Effect of Global Travel and Contact Restrictions on Mitigating the COVID-19 Pandemic. Engineering.

Lai, S., Sorichetta, A., Bondarenko, M., 2020b. Global Public and School Holidays 20102019.

Li, Y., Campbell, H., Kulkarni, D., Harpur, A., Nundy, M., Wang, X., Nair, H., for COVID, U.N., 2021. The temporal association of introducing and lifting non-pharmaceutical interventions with the time-varying reproduction number (R) of SARS-CoV-2: a modelling study across 131 countries. The Lancet Infectious Diseases 21, 193-202.

Lai, S., Ruktanonchai, N.W., Zhou, L., Prosper, O., Tatem, A.J., 2020a. Effect of nonpharmaceutical interventions to contain COVID-19 in China. Nature 1-7.

Moore, S., Hill, E.M., Tildesley, M.J., Dyson, L., Keeling, M.J., 2021. Vaccination and non-pharmaceutical interventions for COVID-19: a mathematical modelling study. Lancet. Infect. Dis 21, 793-802.

Nations, U., 2019. World population prospects 2019: highlights. Department of Economic and Social Affairs, Population Division.

Nguimkeu, P., Tadadjeu, S., 2021. Why is the number of COVID-19 cases lower than expected in Sub-Saharan Africa? A cross-sectional analysis of the role of demographic and geographic factors. World Dev. 138, 105251.

Pang, X., Ren, L., Wu, S., Ma, W., Yang, J., Di, L., Li, J., Xiao, Y., Kang, L., Du, S., 2020. Cold-chain food contamination as the possible origin of COVID-19 resurgence in Beijing. Natl. Sci. Rev. 7, 1861-1864.

Perra, N., 2021. Non-pharmaceutical interventions during the COVID-19 pandemic: A review. Phys. Rep. 23, 1-52.

Petherick, A., Goldszmidt, R.G., Andrade, E.B., Furst, R., Pott, A., Wood, A., 2021. A worldwide assessment of COVID-19 pandemic-policy fatigue. Available at SSRN 3774252.

Prevention, C.f.D.C.a., 2021. COVID-19 Response. COVID-19 Case Surveillance Public Data Access, Summary, and Limitations.

Rodell, M., Houser, P., Jambor, U., Gottschalck, J., Mitchell, K., Meng, C.-J., Arsenault, K., Cosgrove, B., Radakovich, J., Bosilovich, M., 2004. The global land data assimilation system. Bull. Am. Meteorol. Soc. 85, 381-394.

Saad-Roy, C.M., Morris, S.E., Metcalf, C.J.E., Mina, M.J., Baker, R.E., Farrar, J., Holmes, E.C., Pybus, O.G., Graham, A.L., Levin, S.A., 2021. Epidemiological and 
evolutionary considerations of SARS-CoV-2 vaccine dosing regimes. Science 372 363-370.

Sharma, M., Mindermann, S., Rogers-Smith, C., Leech, G., Snodin, B., Ahuja, J., Sandbrink, J.B., Monrad, J.T., Altman, G., Dhaliwal, G., Finnveden, L., 2021 Understanding the effectiveness of government interventions against the resurgence of COVID-19 in Europe. Nat. Commun. 12 (1), 1-13.

Tian, H., Liu, Y., Li, Y., Wu, C., Chen, B., Kraemer, M., Li, B., Cai, J., Xu, B., Yang, Q., 2020. An investigation of transmission control measures during the first 50 days of the COVID-19 epidemic in China. Science.

Van Bavel, J.J., Baicker, K., Boggio, P.S., Capraro, V., Cichocka, A., Cikara, M., Crockett, M.J., Crum, A.J., Douglas, K.M., Druckman, J.N., 2020. Using social and behavioural science to support COVID-19 pandemic response. Nat. Hum. Behav. 4, 460-471.

Wang, J., Xu, C., 2017. Geodetector: Principle and prospective. Acta Geol Sin-Engl.
Wang, J.F., Zhang, T.L., Fu, B.J., 2016. A measure of spatial stratified heterogeneity. Ecol. Ind. 67, 250-256.

WHO, 2021. Weekly epidemiological update on COVID-19 - 23 March 2021, https:// www.who.int/publications/m/item/weekly-epidemiological-update-on-covid-1923-march-2021.

Yang, J., Marziano, V., Deng, X., Guzzetta, G., Zhang, J., Trentini, F., Cai, J., Poletti, P., Zheng, W., Wang, W., 2021. Despite vaccination, China needs non-pharmaceutical interventions to prevent widespread outbreaks of COVID-19 in 2021. Nat. Hum. Behav. 1-12.

Zhou, Q., 2020. International collaboration for global accessibility of COVID-19 vaccines. Natl. Sci. Rev. 7, 1269.

Zhang, W.B., Ge, Y., Liu, M., Atkinson, P.M., Wang, J., Zhang, X., Tian, Z., 2021. Risk assessment of the step-by-step return-to-work policy in Beijing following the COVID19 epidemic peak. Stoch. Env. Res. Risk Assess. 35 (2), 481-498. 